\title{
Understanding the Residual Strain Distribution as a Function of Depth in Alumina/ Stainless Steel Brazing Joint
}

\author{
Chun Li \\ Harbin Institute of Technology, Harbin, China, People's Republic of; \\ chun.li@hit.edu.cn
}

\begin{abstract}
We reveal the novel result about the residual strain distribution as a function of depth in alumina/ stainless steel brazing joint using synchrotron XRD in transmission geometry. The evolution of the residual strain distribution in the joint during cooling is in-situ measured using an in-house developed brazing equipment. The residual strain in the joint is low at relatively high temperature and with the decrease of the temperature, the residual strain increases and becomes compressive. The residual strain is found to be increasing from the surface to the interface in a nonlinear fashion. The microstructure of the joint is characterised and the effect of the pores and the fillet on the residual strain distribution is studied by image based modelling.
\end{abstract}

Keywords: Alumina, Stainless steel, Residual stress, image based modelling, XRD 